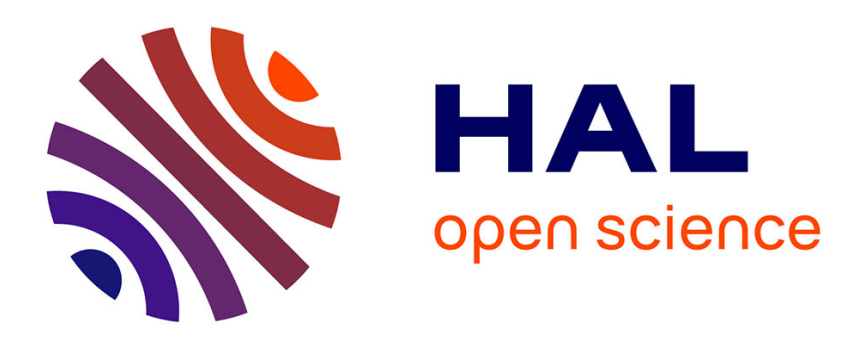

\title{
Influence of the initial phase profile on the asymptotic self-similar parabolic dynamics
}

Christophe Finot, Lei Wu

\section{To cite this version:}

Christophe Finot, Lei Wu. Influence of the initial phase profile on the asymptotic self-similar parabolic dynamics. Journal of Nonlinear Optical Physics and Materials, 2010, 18 (4), pp.709-721. 10.1142/S0218863509004944 . hal-00469842

\section{HAL Id: hal-00469842 \\ https://hal.science/hal-00469842}

Submitted on 4 Apr 2010

HAL is a multi-disciplinary open access archive for the deposit and dissemination of scientific research documents, whether they are published or not. The documents may come from teaching and research institutions in France or abroad, or from public or private research centers.
L'archive ouverte pluridisciplinaire HAL, est destinée au dépôt et à la diffusion de documents scientifiques de niveau recherche, publiés ou non, émanant des établissements d'enseignement et de recherche français ou étrangers, des laboratoires publics ou privés. 


\title{
Influence of the initial phase profile on the asymptotic self-similar parabolic dynamics
}

\author{
Christophe FINOT $^{1}$, Lei WU $^{2}$ \\ ${ }^{1}$ Institut CARNOT de Bourgogne, UMR 5029 CNRS-Université de Bourgogne, Dijon, \\ FRANCE \\ ${ }^{2}$ Tianmu College, Zhejiang Forestry University, Li'an 311300, CHINA \\ christophe.finot@u-bourgogne.fr
}

(Received xx August 2009; final version received $x$ August 2009)

\begin{abstract}
We describe the influence of the initial phase profile on the convergence towards asymptotic self-similar parabolic shape. More precisely, based on numerical simulations, we discuss the impact of an initial linear chirp and a $\pi$ phase shift. If the parabolic shape has been found to describe accurately the pulse envelope, dark structures can appear and evolve also self-similarly on the parabolic background.
\end{abstract}

Keywords: Parabolic pulses, nonlinear propagation, nonlinear fibre

\section{Introduction}

Since their first experimental demonstration in 2000 in normally dispersive fiber amplifiers [1], the unique properties of optical parabolic pulses have generated a great deal of attention. Indeed, under the combined influence of optical gain, dispersion and Kerr nonlinearity, any pulse progressively evolves towards a parabolic intensity profile combined with a strictly linear chirp [1-3]. This typical shape experiences then a self-similar evolution, with exponential spectral and temporal broadenings and remarkably resists to the deleterious effects of the optical wave-breaking $[4,5]$. Such features have already stimulated various applications [6], in the fields of ultra-short high-power pulse generation $[1,3,7,8]$ and optical telecommunications $[5,9,10]$. Experimental demonstrations based on rare earth doped fibers (with ytterbium [1, 7] or erbium [3, 8] dopants) or on Raman distributed amplifiers [9-11] have indeed been successfully achieved. 
One the most fascinating features that the theoretical and experimental works have reported is that any pulse, irrespective to its initial shape, progressively reshapes into an asymptotic profile $[1-3,12]$ only determined by the initial pulse energy. In other words, the parabolic profile behaves as an attractor of the system, which is very attractive for practical applications such as pulse shaping [9] or optical regeneration [10]. The observation of such a behavior has also been recently extended to other nonlinear optical systems that support asymptotic parabolic propagation such as graded-index waveguide amplifiers [13] or $(3 \mathrm{D}+1)$ spatio-temporal light bullets [14]. To date, the study of this remarkable property in fibers has essentially been focused on the impact of the temporal intensity profile, with theoretical works investigating the consequences of various symmetric initial pulse shapes [15] or temporal durations $[1-3,12]$. The influence of the initial phase profile has never been specifically investigated, even if it has already been suggested that an initial linear chirp could change the dynamics experienced in an erbium-doped fiber amplifier [8]. In this article, we try to address this lack by considering successively the impact of a linear chirp and a $\pi$ phase shift on a Gaussian pulse evolving in a fiber amplifier. We will show that the transient evolution towards the asymptotic parabolic profile strongly depends on the initial phase profile and we will check that the envelope of the amplified pulse is well described by the asymptotic self-similar solution. However, we will also highlight that dark structures can exist and evolve on the parabolic background.

This article is thus organized as follows. We first describe the model we use, the asymptotic pulse properties and the various parameters chosen to characterize the convergence towards the asymptotic state. In order to illustrate this section, we first base our discussion on the influence of the initial temporal width. We then focus on 
the dynamics of an initial linearly-chirped pulse and we outline the role of the initial chirp coefficient value. Finally, the consequences of an initial $\pi$ phase shift are discussed.

\section{Numerical Model and parameters used to describe the pulse dynamics}

\subsection{NLSE with gain and asymptotic solution}

The evolution of the complex electrical field $\psi(z, T)$ of a pulse propagating in a fiber amplifier can be described by the well-known scalar nonlinear Schrödinger equation (NLSE) with gain [16] :

$$
i \frac{\partial \psi}{\partial z}=i \frac{g}{2} \psi+\frac{\beta_{2}}{2} \frac{\partial^{2} \psi}{\partial T^{2}}-\gamma|\psi|^{2} \psi
$$

with $z$ the propagation distance and $T$ the time in a copropagating frame. $\gamma$ is the nonlinear Kerr coefficient, $\beta_{2}$ is the second order dispersion and $g$ is the gain that is here assumed to be constant. For the sake of simplicity, we do not include in the present discussion the effects of higher-order linear and nonlinear effects such as third order dispersion, linear attenuation or intrapulse Raman scattering.

Techniques based on the asymptotic self-similar analysis of this equation have outlined that, in the normal regime of dispersion, the evolution of any pulse can be accurately described in the limit $z \rightarrow \infty$ by the following asymptotic complex solution $\psi_{P}(z, T)[1]:$

$$
\begin{cases}\psi_{P}(z, T)=A_{P}(z) \sqrt{1-\frac{T^{2}}{T_{P}^{2}(z)}} \exp \left(-i \frac{C_{P}}{2} T^{2}+i \varphi_{P}\right) & \text { if }|T| \leq T_{P} \\ \psi_{P}(z, T)=0 & \text { otherwise }\end{cases}
$$


This solution corresponds to a compactly-supported pulse with a parabolic intensity profile whose zero-crossing points are given by $T_{P}(z) . A_{P}(z), C_{P}$ and $\varphi_{P}$ are the amplitude of the pulse, the linear chirp slope and a constant phase offset, respectively. Their longitudinal evolution are explicitly given by the following set of equations $[1,17,18]$ :

$$
\left\{\begin{array}{l}
A_{P}(z)=\frac{1}{2} E_{0}^{1 / 3}\left(\frac{g}{\sqrt{\beta_{2} \gamma / 2}}\right)^{1 / 3} \exp \left(\frac{g}{3} z\right) \\
T_{P}(z)=3 E_{0}^{1 / 3} \frac{\sqrt[3]{\beta_{2} \gamma / 2}}{g^{2 / 3}} \exp \left(\frac{g}{3} z\right) \\
C_{P}=\frac{g}{3 \beta_{2}} \\
\varphi_{P}=\frac{3 \gamma A_{o}^{2}}{2 g} \exp \left(\frac{2 g z}{3}\right)
\end{array}\right.
$$

with $E_{0}$ being the initial pulse energy. In the frequency domain, the corresponding asymptotic power spectrum is also parabolic with zero-crossing points of $\pm v_{p}(z)$ where $v_{P}(z)=C_{P} T_{P}(z)$. The corresponding root-mean square (rms) temporal and spectral widths are respectively [12] :

$$
T_{\mathrm{Prms}}=\frac{2 T_{P}}{\sqrt{5}} \quad \text { and } \quad v_{\mathrm{Prms}}=\frac{2 v_{P}}{\sqrt{5}}
$$

Let us note that a physical gain is not a mandatory requirement and that a similar dynamics can also be observed in passive fibers where a continuously tapered dispersion profile mimics the consequences of the gain [19]. Our conclusions could therefore be straightforwardly extended from fiber amplifiers to passive devices using such dispersion management [20-22].

In order to illustrate those analytical predictions and our discussions, we numerically solve Eq. (1) by symmetric split-step Fourier method [16]. We consider the propagation in an existing Raman amplifier [11] based on a normally dispersive 
fiber with $\beta_{2}=16.610^{-3} \mathrm{ps}^{2} / \mathrm{m}$ and $\gamma=4 \mathrm{~W}^{-1} \cdot \mathrm{km}^{-1}$. The gain of the device is $1.8 \mathrm{~dB} / \mathrm{km}$. The initial pulses $\psi_{0}(T)$ are Gaussian pulses with an energy $E_{0}$ of $6 \mathrm{pJ}$, an initial rms temporal width $T_{G}$ (which corresponds to a full-width at half-maximum (FWHM) of $\sqrt{2 \ln 2} T_{G}$ ) and with an initial phase profile $\varphi_{0}(T)$ :

$$
\psi_{G}(0, T)=\sqrt{\frac{E_{0}}{T_{G}}}\left(\frac{2}{\pi}\right)^{\frac{1}{4}} \quad \exp \left(-\frac{T^{2}}{T_{G}^{2}}\right) \quad \exp \left(i \varphi_{0}(T)\right)
$$

In the present work, we specifically investigate several initial phase profiles $\varphi_{0}(T)$ corresponding to linear chirps and to $\pi$-phase shifts. More explicitly, the linear chirp (Figure 1(a1)) is described by an initial parabolic phase :

$$
\varphi_{0}(T)=-\frac{C_{0}}{2} T^{2}
$$

with $C_{0}$ being the chirp coefficient, i.e. the slope of the linear chirp. We will test both positive and negative values of $C_{0}$ (leading to normal and anomalous chirps respectively). Regarding the profiles having a phase shift of $P$ rad centered at $T=T_{C}$, in order to have realistic parameters, we have taken into account a transition having an hyperbolic-tangent profile with a characteristic duration $T_{S}$ (Figure 1(b1)) :

$$
\varphi_{0}(T)=-\frac{P}{2}\left(1-\tanh \left(\frac{T-T_{C}}{T_{S}}\right)\right)
$$

\subsection{Parameters used to investigate the pulse dynamics}

In order to introduce different useful parameters, let us first recall, as a pedagogical example, the already-studied problem of the influence of the initial pulse duration. We consider Fourier-limited initial pulses (i.e. $\varphi_{0}(T)=0$ ) with temporal FWHM ranging from 5 ps to 150 ps. The longitudinal evolution of the rms temporal width during amplification is plotted on a semi-logarithmic scale in Figure 2(a). After a given length of propagation in the distributed amplifier, all the pulses experience the same 
exponential broadening that is found in excellent agreement with the analytical predictions of Eq. (3) $[1-3,12]$. The spectral evolution of the pulses (Figure 2(b)) follows a similar expansion and despite initial temporal widths varying by a factor 30 , all the pulses ultimately follow the same longitudinal trends.

An interesting additional way to visualize simultaneously the temporal and spectral evolution towards the asymptotic state (Figure 1(c)) is the phase portrait in the time-frequency plane with the temporal and spectral widths ( $T_{r m s}$ and $v_{r m s}$ respectively) normalized with respect to the analytical predictions given at the same distance by Eqs. (3) and (4) $[3,12,14]$ : spiral trajectories of the different pulses all converge towards the central point, clearly highlighting the attractive nature of the asymptotic solution.

In order to better quantity this spectro-temporal convergence towards the asymptotic properties, we introduce a parameter we call distance factor $D$ defined as the separation between the normalized temporal and spectral rms widths and the corresponding asymptotic properties defined by Eq. (3-4). This parameter is therefore defined as :

$$
D=\sqrt{\left(\frac{T_{\text {rms }}}{T_{\text {Prms }}}-1\right)^{2}+\left(\frac{v_{\text {rms }}}{v_{\text {Prms }}}-1\right)^{2}}
$$

From Figure 3(a), all the pulses progressively evolve towards $D=0$, which clearly reflects that their temporal and spectral parameters converge towards the asymptotic predictions. We can also make out that the propagation distance over which the convergence is achieved significantly depends on the initial temporal duration $[1,2,12,23,24]$.

Despite their interest, neither the Distance factor $D$ nor the plots of Figure (2) are fully sufficient to definitively conclude on the convergence towards a parabolic asymptotic state. Indeed, those elements do only provide insights of the temporal and 
spectral widths of the pulse and do not provide any information on its actual shape. It is therefore required to use an additional parameter to characterize more precisely the temporal intensity profile. Potential solutions rely on higher-order moments such as kurtosis [25], or on other dimensionless functionals [26]. We prefer to choose a misfit parameter defined as the difference between the intensity profile of the pulse and the intensity profile of a compact parabolic intensity profile $I_{P}[24,27,28]$ :

$$
M^{2}=\int\left[|\psi|^{2}-I_{P}\right]^{2} d t / \int|\psi|^{4} d t
$$

This misfit parameter enables the direct comparison of the pulse shape with the parabolic ideal shape. We can therefore check on Figure 3(b) that irrespective to their initial duration, all the pulses converge towards a misfit parameter with a null value, which is synonym of a parabolic intensity profile. We can however note here, that compared to the evolution of the $D$ factor which decreases nearly monotonously down to zero, the misfit factor evolution can be much less monotonous with local minima, which has already been shown previously [29]. Let us finally note that we could have included both the $D$ and $M$ factors in a single indicator which would have been a misfit factor relative to a parabolic intensity profile having the parameters as defined by Eq. (3). We have however preferred two indicators, in order to discuss separately the influence of the shape and the influence of the temporal/spectral evolution.

\section{Influence of an initial linear chirp}

We now study the influence of the initial phase and more specifically, the influence of an initial linear chirp as described by Eq. (6). We consider initial 80-ps Gaussian pulses with a chirp coefficient $C_{0}$ ranging from -0.01 rad.ps ${ }^{-2}$ to 0.01 rad.ps ${ }^{-2}$ (Figure 1(a1)) The longitudinal temporal and spectral evolutions plotted on Figure 4(a-b) 
confirm the role of attractor played by the self-similar solution. Indeed, whatever the initial chirp coefficient value or sign are, the pulse properties quantatively converge towards the evolution predicted by Eq. (4-5). However, the paths towards the asymptotic sink differ markedly depending on the initial sign of the chirp coefficient, as can be noticed in Figure 4(c) : whereas the starting point does not depend on the chirp sign, the trajectory is fundamentally different.

Pulses with an initial normal chirp (i.e. $C_{0}>0$ ) undergo a continuous increase of their temporal and spectral widths and follow a traditional spiral trajectory. By contrast, the evolution of pulses having an anomalous chirp is a bit more complex. Indeed, those pulses undergo an initial stage of temporal compression linked to the compensation of the initial chirp by normal dispersion [16]. But this stage of temporal compression is not a purely linear chirp compensation, as highlighted by the simultaneous changes observed in the spectrum of the pulses. To be more precise, the pulses also experience a spectral compression, which is typical of pulses with initial anomalous chirp propagating in a nonlinear Kerr medium [24, 30, 31]. Remark that at a given stage of propagation (points A or B), the pulses are close from transformlimited pulses (time-bandwidth product of 0.6).

If now we consider the evolutions of the $D$ and $M$ factors, we can notice that the evolution of pulses having an anomalous initial chirp is more complex than pulses with a normal chirp. We can once again see the particular points $\mathrm{A}$ and $\mathrm{B}$ corresponding to turning points in the evolution of the pulse. We could have anticipated from a somewhat naive approach that an initial normal chirp being closer from the asymptotic solution would converge faster towards the asymptotic solution. However, quite counterintuitively, pulses with initial anomalous chirp converge faster that their normal counterpart. 
From the Figure 5(a), we can see that after $25 \mathrm{~km}$, the resulting misfit factor is slightly higher for pulses with initial normal dispersion. In order to get further insight, we have plotted the corresponding intensity profiles obtained after $25 \mathrm{~km}$ (Figure 6(a)). As we it can be seen, all the pulses, even with very different initial properties, have acquired a parabolic envelope. But if we pay more attention to the details (see Figure 6(b)), it turns out that some ultrashort fluctuations on the parabolic background can be noticed. Those non-sinusoidal oscillations are marks of a stage of propagation where the wave-breaking phenomenon initiates oscillations in the temporal wings of the pulse [29, 32]. Previous works have indeed stressed that an initial chirp could strengthen the wake-breaking impact [33]. Then, under the combined influence of the adiabatic gain and the nonlinearity, those structures evolve towards dark solitons [3436], as confirmed by the excellent agreement between the shape of one of this dip of light and the typical shape of a grey soliton [16, 37] (Figure 6(b)).

\section{Evolution of an initial phase defect}

We now examine the evolution of a pulse exhibiting a phase shift such as described by Eq. (7) (Figure. 1(b)). This phase shift is located at $T_{C}=30$ ps. From Figure 7(a), we can make out that the rms temporal width evolution of the pulse is not very sensitive to the initial pulse shift. On the contrary, the spectral evolution is much more affected (Figure 7(b)), with an initial pulse having a much broader spectral extension. We can also note that a phase shift of $\pi$ or $-\pi$ does not lead to identical evolutions. However, in both cases, the phase portrait exhibits the signature of a rapid convergence toward the asymptotic features.

From the evolution of the distance factor $D$, it could appear that the initial phase shift has only a moderate influence on the propagation (Figure 8(a)). However, 
the misfit factor $M$ (Figure 8(b)) stresses that the initial phase defect deeply influences the pulse shape evolution. Indeed, the associated misfit factor is much higher than those obtained in the case of a chirp-free pulse. This can be better understood when looking at the intermediate pulse temporal intensity profile at $L=5 \mathrm{~km}$ where we can clearly visualize that the initial phase shifts have severely impaired the pulse dynamics, leading to deeply asymmetric profiles (Figure 8(c)). This arises from the fact that during the propagation, the phase defect has translated into an intensity change through the action of dispersion. Despite this highly distorted profile, the misfit factor ultimately decreases down to a low value, confirming the evolution towards a parabolic profile.

From the analysis of $M$ and $D$ factors, one may have concluded that the output pulse is fully described by Eqs. (3-5). Figure 9(a1) depicts the output amplified intensity profile after $20 \mathrm{~km}$ : the global shape is clearly in good agreement with a compact parabolic function. However, if we look more closely into the details, we can also make out the presence of several dips of light on the parabolic background. Those dark structures are spontaneously formed during the propagation and result directly from the initial phase shift (similar examples have also been outlined in other fields such as Bose-Einstein condensate [38, 39]). Once again, their intensity profile has been found in excellent agreement with the analytical expression of a grey soliton (Figure 9(a2)) and we have carefully checked that they do not constitute a transient state : once formed, they evolve on the parabolic background, experiencing a progressive temporal narrowing when the peak power increases. Analytical approximate but highly accurate formula predicting the evolution of those systems have already been proposed in the context of self-similar dark similaritons evolving in 
nonlinear index graded waveguide amplifiers [40,41] or in fibers with a precise longitudinally dispersion varying profile [42].

From Figure 9(b), we can also make out that the sign of the initial phase shift influences the evolution. Indeed, for the $\pi$ phase shift, the temporal chirp resulting from the finite transition time is of opposite sign as the intensity gradient and therefore is of the same sign as the chirp induced by self-phase modulation. As a result, the resulting dark structure will be first repulsed (Figure 9(b1)). On the contrary, for a $-\pi$ phase shift, the first stage of propagation will be attractive towards the center of the pulse (Figure 9(b2)).

\section{Conclusions}

We have investigated in this work for the first time of our knowledge the influence of the initial phase profile on the convergence towards the asymptotic parabolic state in a distributed fiber amplifier with normal dispersion. We have shown that the asymptotic solution describes accurately the evolution of the rms temporal and spectral widths and that the temporal intensity profile is asymptotically in good agreement with the expected parabolic profile, irrespective to the initial phase profile of the pulse. However, the details of the asymptotically obtained pulses reveal some sensitivity to phase defects with the existence of dark ultrashort solitonic structures that are not predicted by the asymptotic solution and that can further maintain during amplification. Their central position evolves on the parabolic background and they experience temporal narrowing during the amplification. 


\section{References}


1. M. E. Fermann, V. I. Kruglov, B. C. Thomsen, J. M. Dudley, and J. D. Harvey, "Self-similar propagation and amplification of parabolic pulses in optical fibers," Phys. Rev. Lett. 84, 6010-6013 (2000).

2. C. Finot, G. Millot, and J. M. Dudley, "Asymptotic characteristics of parabolic similariton pulses in optical fiber amplifiers," Opt. Lett. 29, 2533-2535 (2004).

3. J. M. Dudley, C. Finot, G. Millot, and D. J. Richardson, "Self-similarity in ultrafast nonlinear optics," Nat. Phys. 3, 597-603 (2007).

4. D. Anderson, M. Desaix, M. Karlson, M. Lisak, and M. L. QuirogaTeixeiro, "Wave-breaking-free pulses in nonlinear optical fibers," J. Opt. Soc. Am. B 10, 1185-1190 (1993).

5. F. Parmigiani, C. Finot, K. Mukasa, M. Ibsen, M. A. F. Roelens, P. Petropoulos, and D. J. Richardson, "Ultra-flat SPM-broadened spectra in a highly nonlinear fiber using parabolic pulses formed in a fiber Bragg grating," Opt. Express 14, 7617-7622 (2006).

6. C. Finot, J. M. Dudley, B. Kibler, D. J. Richardson, and G. Millot, "Optical parabolic pulse generation and applications," IEEE J. Quantum Electron. in press(2009).

7. P. Dupriez, C. Finot, A. Malinowski, J. K. Sahu, J. Nilsson, D. J. Richardson, K. G. Wilcox, H. D. Foreman, and A. C. Tropper, "Highpower, high repetition rate picosecond and femtosecond sources based on Yb-doped fiber amplification of VECSELS," Opt. Express 14, 9611-9616 (2006).

8. J. W. Nicholson, A. Yablon, P. S. Westbrook, K. S. Feder, and M. F. Yan, "High power, single mode, all-fiber source of femtosecond pulses at 1550 nm and its use in supercontinuum generation," Opt. Express 12, 30253034 (2004).

9. C. Finot and G. Millot, "Synthesis of optical pulses by use of similaritons," Opt. Express 12, 5104-5109 (2004).

10. C. Finot, S. Pitois, and G. Millot, "Regenerative 40-Gb/s wavelength converter based on similariton generation," Opt. Lett. 30, 1776-1778 (2005).

11. K. Hammani, C. Finot, S. Pitois, J. Fatome, and G. Millot, "Real time measurement of long parabolic optical similaritons," Electron. Lett. 44, 1239-1240 (2008).

12. S. Wabnitz, "Analytical Dynamics of parabolic pulses in nonlinear optical fiber amplifiers," IEEE Photon. Technol. Lett. 19, 507-509 (2007).

13. L. Wu, L. Li, and J.-F. Zhang, "Controllable generation and propagation of asymptotic parabolic optical waves in graded-index waveguide amplifiers," Phys. Rev. A 78(2008). 
14. S. Chen and J. M. Dudley, "Spatiotemporal nonlinear optical selfsimilarity in three dimensions," Phys. Rev. Lett. 102, 233903 (2009).

15. V. I. Kruglov and J. D. Harvey, "Asymptotically exact parabolic solutions of the generalized nonlinear Schrödinger equation with varying parameters," J. Opt. Soc. Amer. B 23, 2541-2550 (2006).

16. G. P. Agrawal, Nonlinear Fiber Optics, Third Edition (Academic Press, San Francisco, CA, 2001).

17. V. I. Kruglov, A. C. Peacock, J. M. Dudley, and J. D. Harvey, "Selfsimilar propagation of high-power parabolic pulses in optical fiber amplifiers," Opt. Lett. 25, 1753-1755 (2000).

18. V. I. Kruglov, A. C. Peacock, J. D. Harvey, and J. M. Dudley, "Selfsimilar propagation of parabolic pulses in normal-dispersion fiber amplifiers," J. Opt. Soc. Amer. B 19, 461-469 (2002).

19. T. Hirooka and M. Nakazawa, "Parabolic pulse generation by use of a dispersion-decreasing fiber with normal group-velocity dispersion," Opt. Lett. 29, 498-500 (2004).

20. C. Finot, B. Barviau, G. Millot, A. Guryanov, A. Sysoliatin, and S. Wabnitz, "Parabolic pulse generation with active or passive dispersion decreasing optical fibers," Opt. Express 15, 15824-15835 (2007).

21. B. Kibler, C. Billet, P. A. Lacourt, R. Ferrière, L. Larger, and J. M. Dudley, "Parabolic pulse generation in comb-like profiled dispersion decreasing fibre," Electron. Lett. 42, 965-966 (2006).

22. A. A. Sysoliatin and D. A. Nolan, "Optical signal processing in dispersion varying fiber," J. Nonlinear Opt. Phys. Mater 16, 171-184 (2007).

23. Y. Ozeki, Y. Takushima, K. Taira, and K. Kikuchi, "Clean similariton generation from an initial pulse optimized by the backward propagation method," in Conference on Lasers and Electro-Optics (CLEO US), (OSA Trends in Optics and Photonics Series, 2004), CTuBB51113-51114.

24. C. Finot, F. Parmigiani, P. Petropoulos, and D. J. Richardson, "Parabolic pulse evolution in normally dispersive fiber amplifiers preceding the similariton formation regime," Opt. Express 14, 3161-3170 (2006).

25. C.-K. Rosenberg, D. Anderson, M. Desaix, P. Johannisson, and M. Lisak, "Evolution of optical pulses towards wave breaking in highly nonlinear fibres," Opt. Commun. 273, 272-277 (2007).

26. S. Boscolo, A. I. Latkin, and S. K. Turitsyn, "Passive nonlinear pulse shaping in normally dispersive fiber systems," IEEE J. Quantum Electron. 44, 1196-1203 (2008). 
27. A. C. Peacock, "Self-similar amplification and propagation of parabolic pulses in optical fibers," M.S. thesis (Master thesis at Auckland University, New-Zealand, 2001).

28. C. Finot, L. Provost, P. Petropoulos, and D. J. Richardson, "Parabolic pulse generation through passive nonlinear pulse reshaping in a normally dispersive two segment fiber device," Opt. Express 15, 852-864 (2007).

29. C. Finot, B. Kibler, L. Provost, and S. Wabnitz, "Beneficial impact of wave-breaking or coherent continuum formation in normally dispersive nonlinear fibers," J. Opt. Soc. Am. B 25, 1938-1948 (2008).

30. M. Oberthaler and R. A. Höpfel, "Spectral narrowing of ultrashort laser pulses by self-phase modulation in optical fibers," Appl. Phys. Lett. 63, 1017-1019 (1993).

31. J. P. Limpert, N. Deguil-Robin, I. Manek-Hönninger, F. Salin, T. Schreiber, A. Liem, F. Röser, H. Zellmer, A. Tünnermann, A. Courjaud, C. Hönninger, and E. Mottay, "High-power picosecond fiber amplifer based on nonlinear spectral compression," Opt. Lett. 30, 714-716 (2005).

32. W. J. Tomlinson, R. H. Stolen, and A. M. Johnson, "Optical wavebreaking of pulses in nonlinear optical fibers," Opt. Lett. 10, 457-459 (1985).

33. M. Gregory Forest, J. N. Kutz, and K. R. T. McLaughlin, "Nonsoliton pulse evolution in normally dispersive fibers," J. Opt. Soc. Am. B 16, 1856-1862 (1999).

34. C. Finot and G. Millot, "Interactions of optical similaritons," Opt. Express 13, 5825-5830 (2005).

35. E. M. Dianov, P. V. Mamyshev, A. M. Prokhorov, and S. V. Chernikov, "Generation of a train of fundamental solitons at a high repetition rate in optical fibers," Opt. Lett. 14, 1008-1010 (1989).

36. P. V. Mamyshev, C. Bosshard, and G. I. Stegeman, "Generation of a periodic array of dark spatial solitons in the regime of effective amplification," J. Opt. Soc. Am. B 11, 1254-1260 (1994).

37. Y. S. Kivshar and B. Lutther-Davies, "Dark optical solitons : physics and applications," Phys. Reports 298, 81-197 (1998).

38. S. Burger, K. Bongs, S. Dettmer, W. Ertmer, and K. Sengstock, "Dark solitons in Bose-Einstein condensates," Phys. Rev. Lett. 83, 5198-5201 (1999).

39. S. Stellmer, C. Becker, P. Soltan-Panahi, E.-M. Richter, S. Dörscher, M. Baumert, J. Kronjäger, K. Bongs, and K. Sengstock, "Collisions od dark solitons in elongated bose-einstein condensates," Phys. Rev. Lett. 101, 120406 (2008). 
40. L. Wu, J.-F. Zhang, L. Li, C. Finot, and K. Porsezian, "Similaritons interaction in nonlinear graded-index waveguide amplifiers," Phys. Rev. A 78, 053807 (2008).

41. L. Li, X. Zhao, and Z. Xu, "Dark solitons on an intense parabolic background in nonlinear waveguides," Phys. Rev. A 78, 063833 (2008).

42. L. Wu, J.-F. Zhang, C. Finot, and L. Li, "Propagation of dark similariton on the compact parabolic background in dispersion-managed optical fibers," Opt. Express 17, 8278-8286 (2009). 


\section{Figure Caption}

Figure 1: Temporal phase and intensity profiles of the initial pulses under study (subplots 1 and 2 respectively). The initial pulses are Gaussian pulses with a FWHM width of 80 ps. (a) Pulses with a linear chirp. Chirp coefficients are $0,-0.005,-0.01,0.005,0.01$ rad.ps ${ }^{-2}$ (solid black line, dark grey solid line, light grey solid line, dark grey dashed line, light grey $\begin{array}{ll}\text { dashed line respectively) } & \text { (b) Pulse with a phase shift } P \text { of } \pi \text { and }-\pi \text { centered at }\end{array}$ $T_{C}=30 \mathrm{ps}$ with a characteristic time $T_{S}=7 \mathrm{ps} \quad$ (solid black line and solid grey line respectively). The grey dotted line is the phase of a transformed limited pulse.

Figure 2: Longitudinal evolution of the temporal (a) and spectral (b) rms widths of an initial chirp-free Gaussian pulse evolving in a distributed Raman amplifier. Different initial pulses widths ranging from $5 \mathrm{ps}$ to $150 \mathrm{ps}$ are studied and the results are compared with analytical evolution predicted by Eqs. (3-4). (solid black circles) $\quad$ (c) Phase portrait in the timefrequency plane.

Figure 3 : Longitudinal evolutions of the Distance factor $D$ (a) and the Misfit parameter M (b) for different initial pulse durations.

Figure 4 : Longitudinal evolution of the temporal (a) and spectral (b) rms widths of an initial Gaussian pulse evolving in a distributed Raman amplifier. Different initial chirps (same convention as for Figure 1(a)) are studied and the results are compared with analytical evolution predicted by Eqs. (3-4) (solid black circles). $\quad$ (c) Phase portrait in the timefrequency plane.

Figure 5 : Longitudinal evolutions of the Distance factor $D$ (a) and the Misfit parameter M (b) for different initial chirp coefficients (same convention as for Figure 1(a)).

Figure 6 : Temporal intensity profiles after propagation in $25-\mathrm{km}$ of the Raman amplifier for different initial chirp coefficients (same convention as for Figure 1(a)). Subplot (b) is a magnification of the falling edge of the parabolic profile plotted on subplot (a). Intensity profile of a grey soliton is plotted with filled circles. 
Figure 7 : Longitudinal evolution of the temporal (a) and spectral (b) rms widths of an initial Gaussian pulse evolving in a distributed Raman amplifier. Different initial phase profiles (same convention as for Figure 1(b1)) are studied and the results are compared with analytical evolution predicted by Eqs. (3-4) (solid black circles). $\quad$ (c) Phase portrait in the timefrequency plane.

Figure 8 : Longitudinal evolutions of the Distance factor $D$ (a) and the Misfit parameter M (b) for different initial phase profiles (same convention as for Figure 1(b)). $\quad$ (c) Temporal intensity profiles obtained after a propagation length of $5 \mathrm{~km}$ in the Raman amplifier.

Figure 9: (a) Temporal intensity profiles after propagation in 20-km of the Raman amplifier for different phase profiles (same convention as for Figure 1(b)). Subplot (a2) is a magnification of the falling edge of the parabolic profile plotted on subplot (a). Intensity profile of a grey soliton is plotted with filled black circles. (b) Longitudinal evolution of the temporal intensity profile for pulse having a $\pi$ and $-\pi$ initial phase shifts (subplots (b1) and (b2) respectively). The trajectory of the main grey soliton is highlighted by a dashed line. 

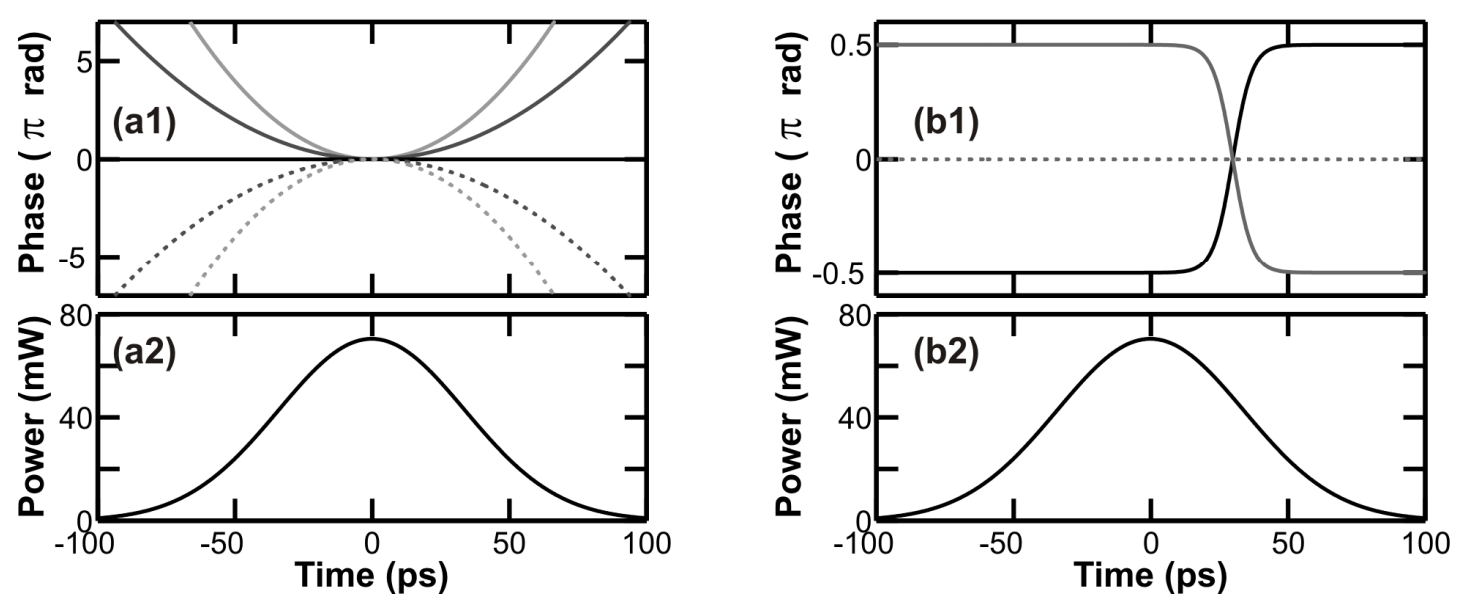

Figure 1

FINOT and WU

Influence of the initial phase profile on the asymptotic self-similar parabolic dynamics 

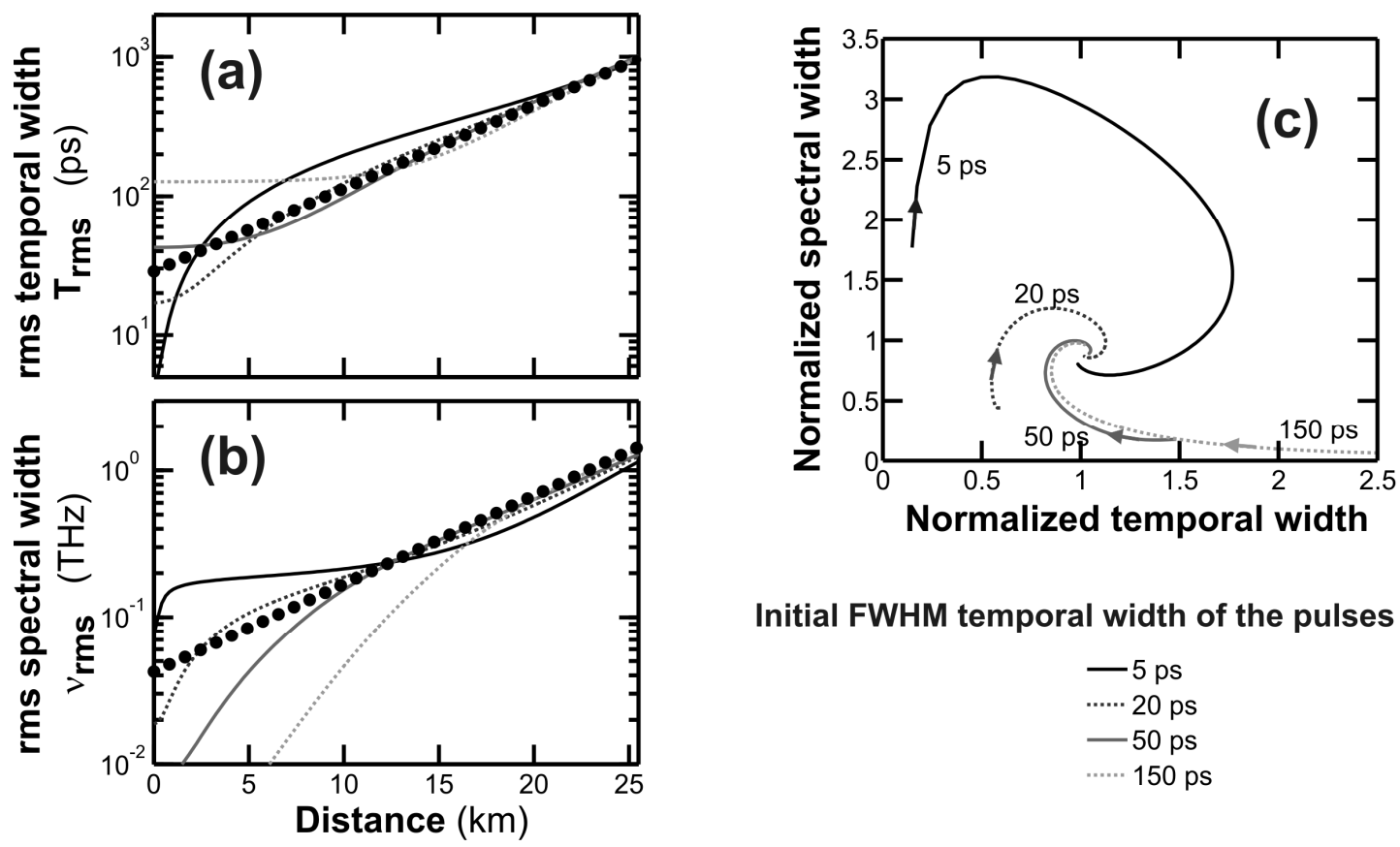

Initial FWHM temporal width of the pulses :

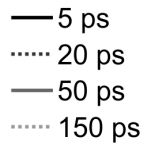

Figure 2

FINOT and WU

Influence of the initial phase profile on the asymptotic self-similar parabolic dynamics 

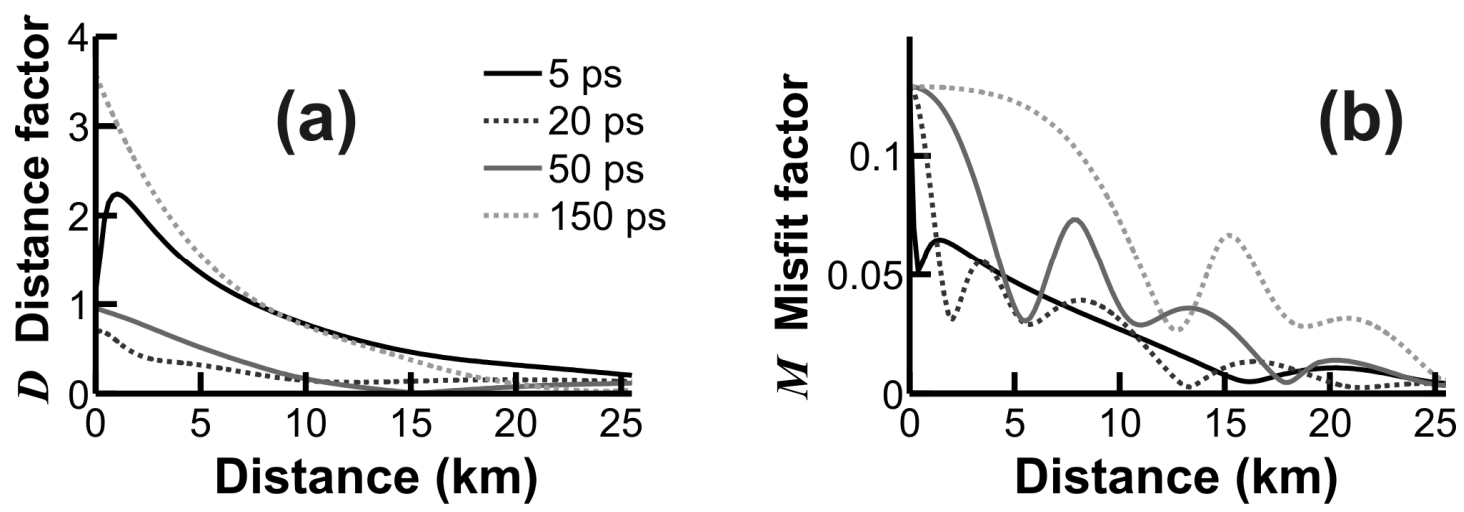

Figure 3

FINOT and WU

Influence of the initial phase profile on the asymptotic self-similar parabolic dynamics 

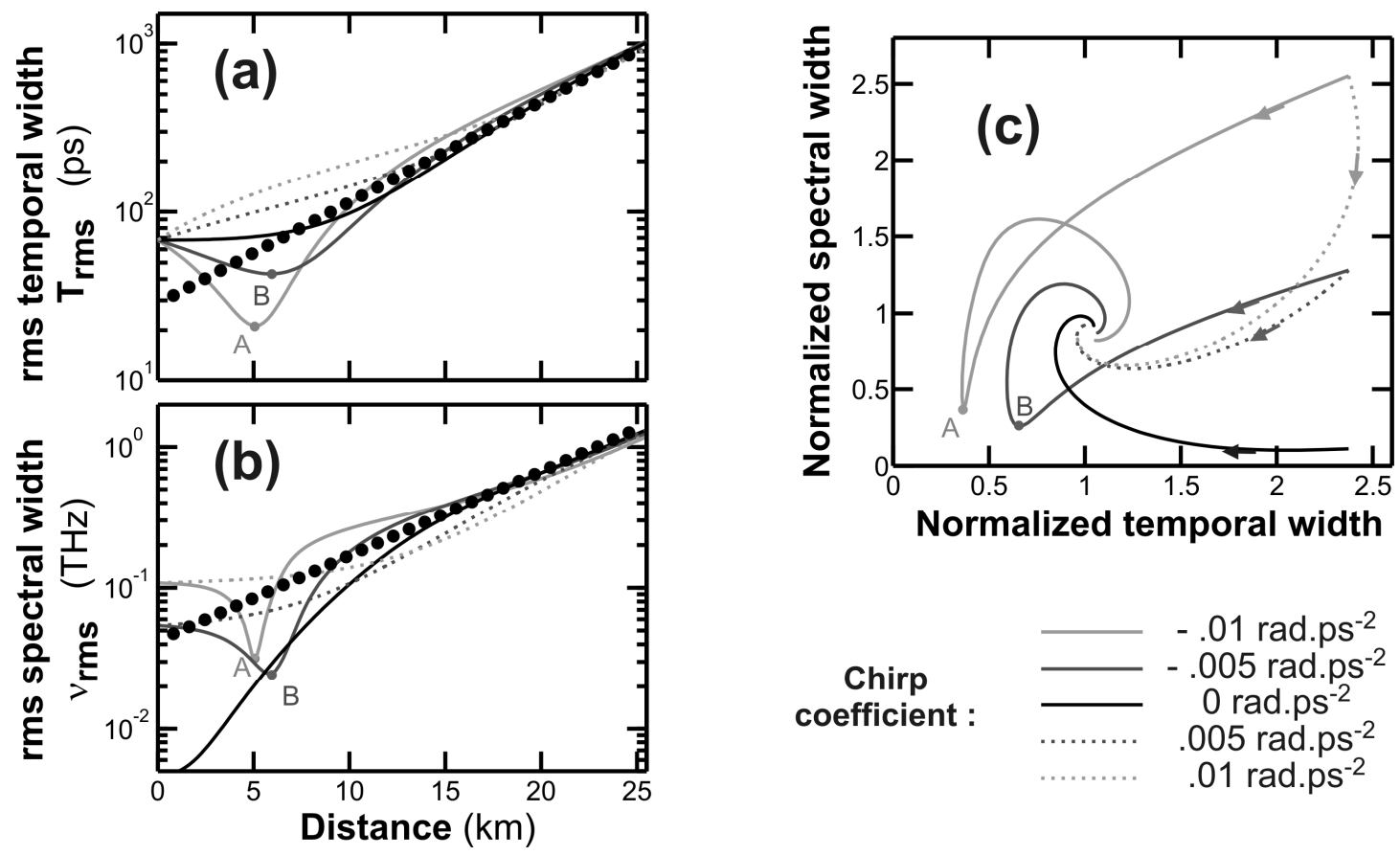

Figure 4

FINOT and WU

Influence of the initial phase profile on the asymptotic self-similar parabolic dynamics 

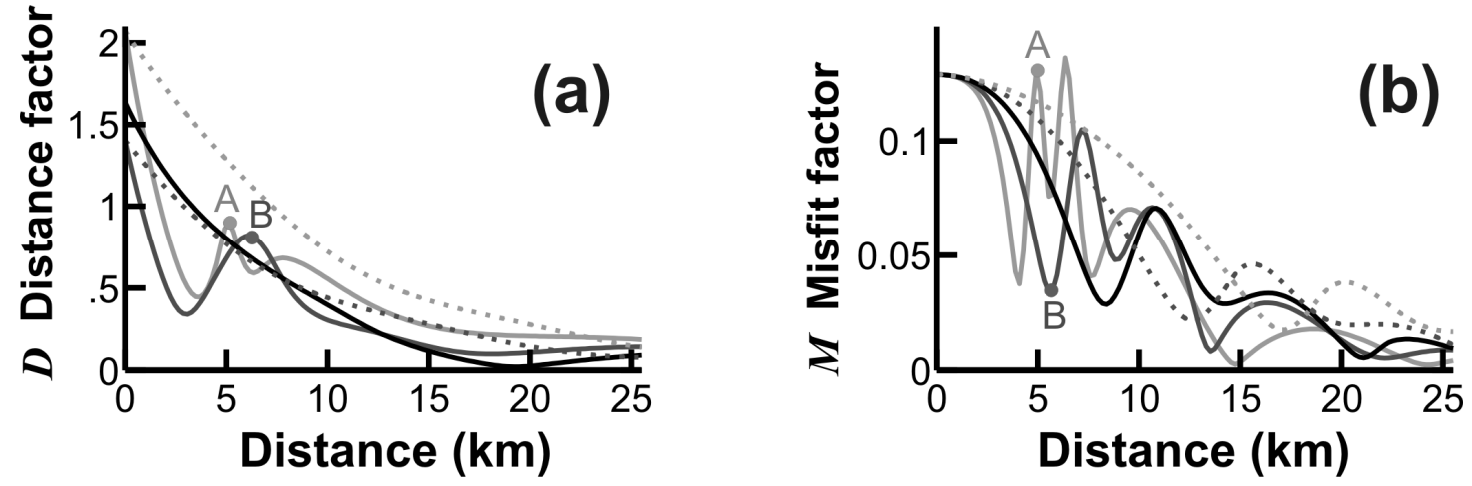

Figure 5

FINOT and WU

Influence of the initial phase profile on the asymptotic self-similar parabolic dynamics 

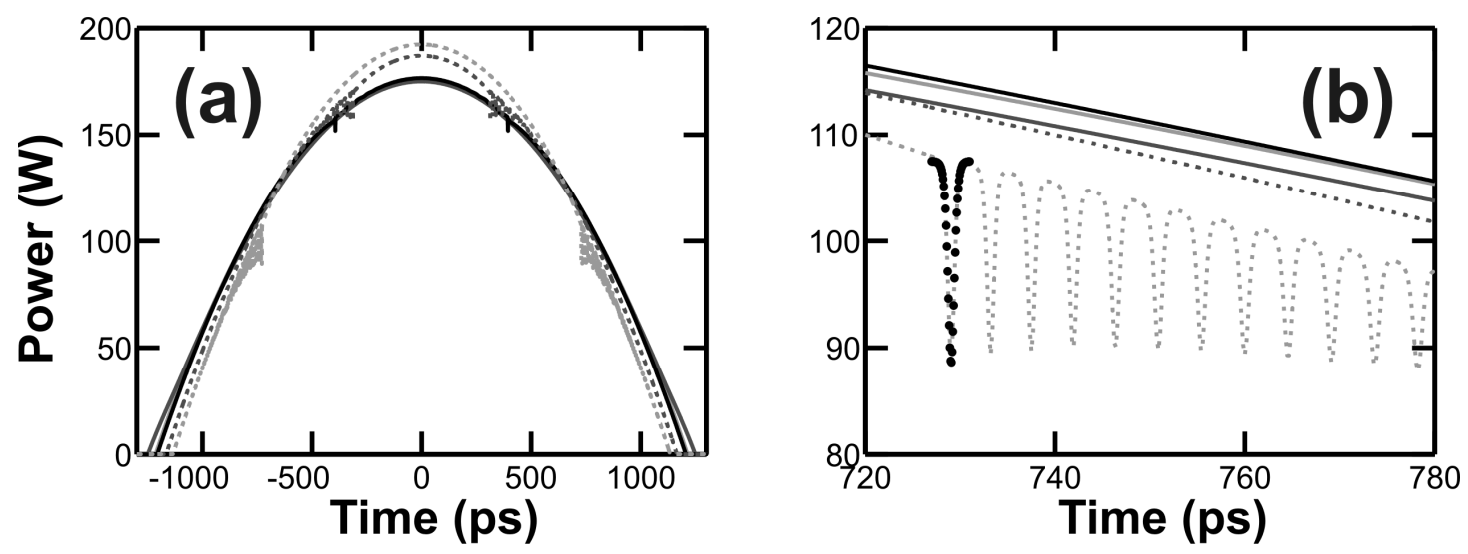

Figure 6

FINOT and WU

Influence of the initial phase profile on the asymptotic self-similar parabolic dynamics 

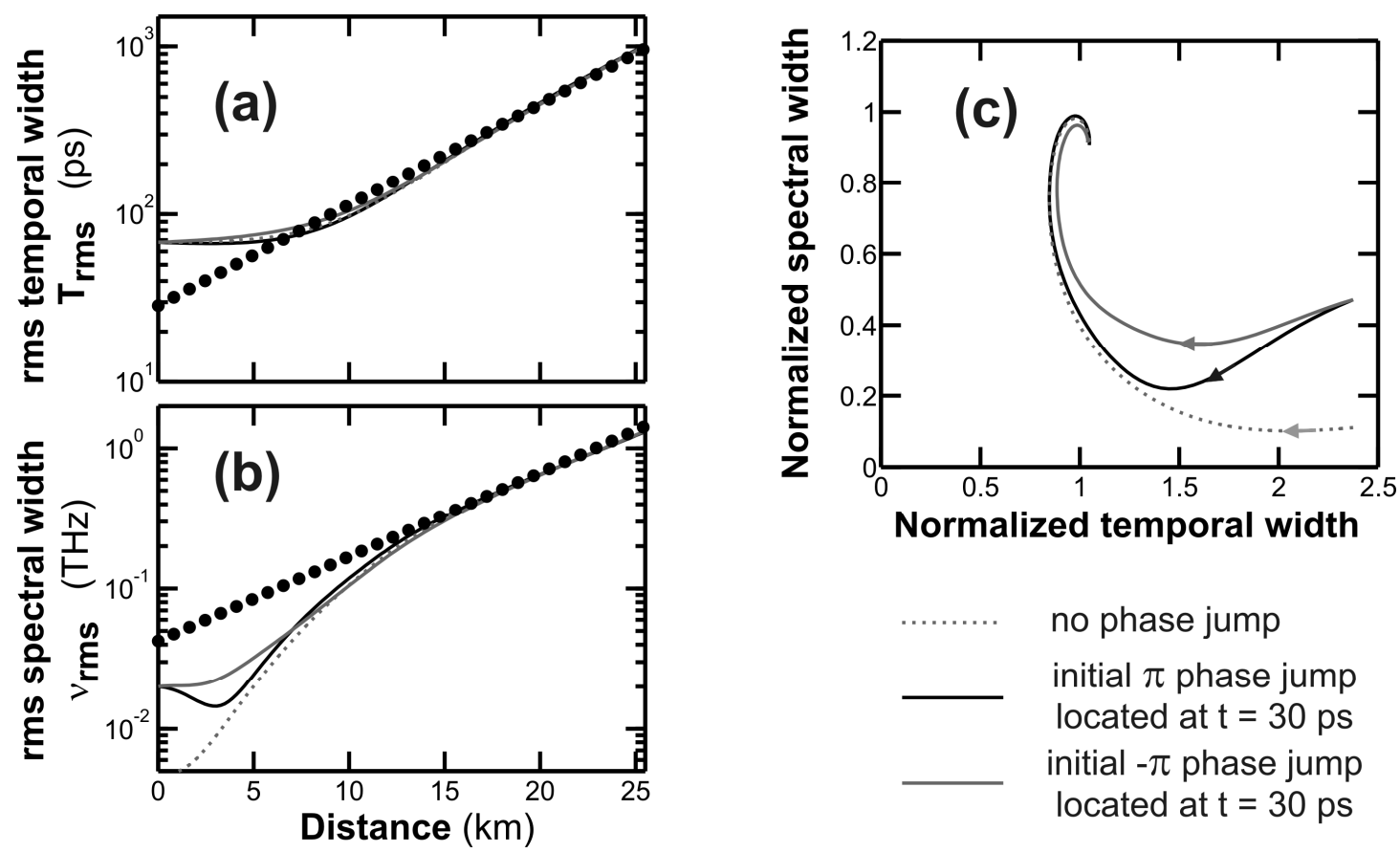

Figure 7

FINOT and WU

Influence of the initial phase profile on the asymptotic self-similar parabolic dynamics 

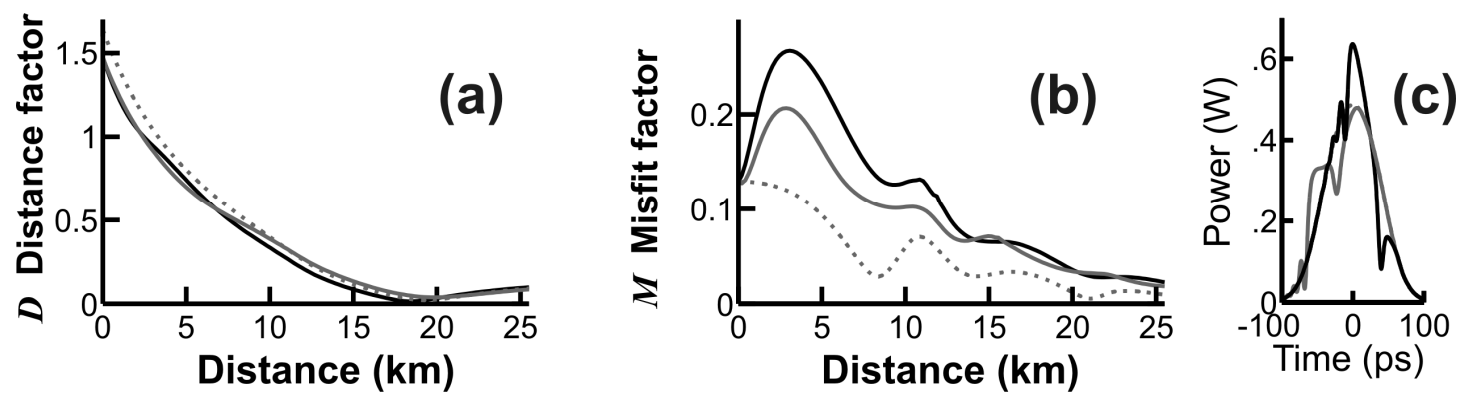

Figure 8

FINOT and WU

Influence of the initial phase profile on the asymptotic self-similar parabolic dynamics 

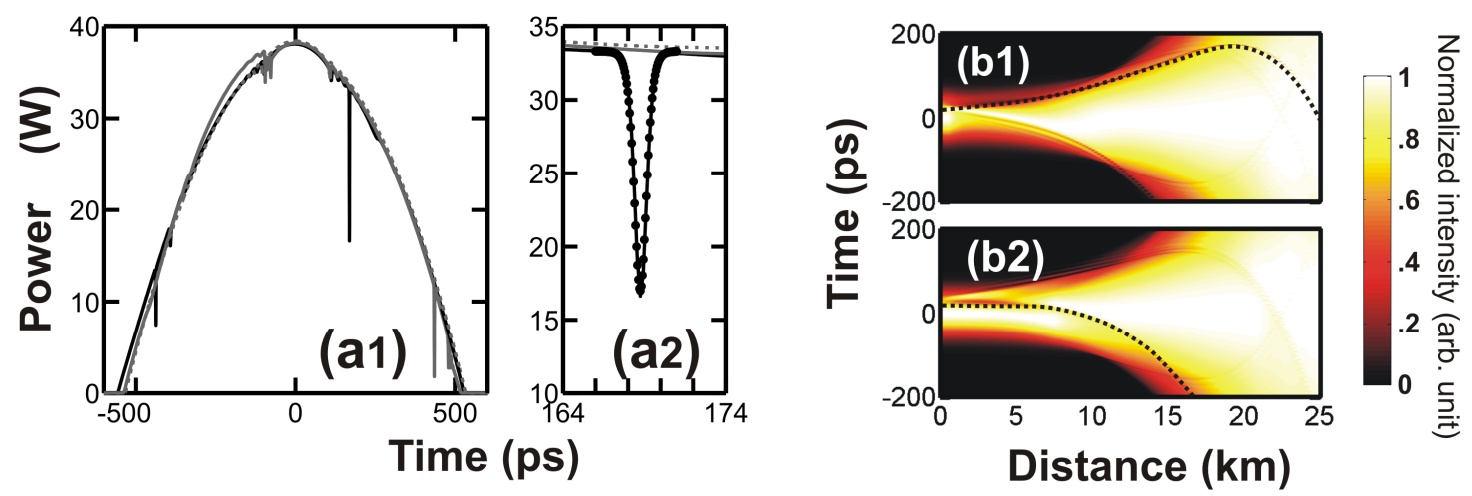

Figure 9

FINOT and WU

Influence of the initial phase profile on the asymptotic self-similar parabolic dynamics 